\title{
Calcário na forma de micropartículas aplicado no sulco de semeadura aumenta produtividade do feijoeiro
}

\author{
Adriano Stephan Nascente ${ }^{1}$ e Tarcísio Cobucci ${ }^{2}$ \\ http://dx.doi.org/10.1590/0034-737X201562060013
}

\section{RESUMO}

A aplicação de solução de micropartículas de calcário $\left(\mathrm{CaCO}_{3}\right)$ no sulco de semeadura pode alterar rapidamente os atributos químicos do solo, na zona de emissão de raízes, e aumentar a produtividade do feijoeiro-comum. O objetivo deste estudo foi o de testar essa hipótese em solos com diferentes características químicas. Foram realizados dois experimentos. No primeiro, avaliaram-se doses de soluções de $\mathrm{CaCO}_{3}(0,1050,2100,4200,8400$ e $\left.12600 \mathrm{~g} \mathrm{ha}^{-1}\right)$, aplicadas no sulco de semeadura, em dois anos agrícolas consecutivos. No segundo, o $\mathrm{CaCO}_{3}(1050$ $\mathrm{g} \mathrm{ha}^{-1}$ ) foi aplicado, ou não, no sulco de semeadura, em quatro ambientes. Os delineamentos experimentais foram em blocos ao acaso. A aplicação de solução de micropartículas de $\mathrm{CaCO}_{3}$, no sulco de semeadura do feijoeiro, aumentou os valores de $\mathrm{pH}$ e reduziu a saturação por $\mathrm{Al}^{+3}$ no solo. Os teores de $\mathrm{Ca}, \mathrm{K}$ e a saturação por bases no solo não foram influenciadas pela adição de $\mathrm{CaCO}_{3}$ no sulco. $\mathrm{O}$ uso de micropartículas de $\mathrm{CaCO}_{3}$ no sulco de semeadura aumentou a produtividade de grãos do feijoeiro-comum. Os resultados sugerem que a aplicação de micropartículas de $\mathrm{CaCO}_{3}$ no sulco de semeadura foi eficiente para alterar os atributos químicos do solo no leito de semeadura e proporcionar incrementos significativos de produtividade de grãos do feijoeiro-comum.

Palavras-chave: Phaseolus vulgaris L., desenvolvimento inicial, correção do solo, carbonato de cálcio, Cerrado.

\section{ABSTRACT}

\section{Lime in microparticulate form applied to the sowing furrow increases common bean yield}

The application of a solution of lime $\left(\mathrm{CaCO}_{3}\right)$ microparticles in the sowing furrow may accelerate the reaction of this corrective in the soil, alter soil chemical attributes and increase common bean (Phaseolus vulgaris L.) yield. The aim of this study was to test this hypothesis in soils with different chemical characteristics. Two experiments were carried out. In the first, it was evaluated solutions with different $\mathrm{CaCO}_{3}$ rates $(0,1050,2100$, $4200,8400 \mathrm{e} 12600 \mathrm{~g} \mathrm{ha}^{-1}$ ) applied to the sowing furrow during two growing seasons. In the second, the solution of $\mathrm{CaCO}_{3}\left(1050 \mathrm{~g} \mathrm{ha}^{-1}\right)$ was applied or not in the sowing furrow in four environments. Both experiments were arranged in a completely randomized block design. The application of a solution of $\mathrm{CaCO}_{3}$ microparticles increased $\mathrm{pH}$ values and reduced the $\mathrm{Al}^{+3}$ saturation in the soil. The concentrations of $\mathrm{Ca}, \mathrm{K}$ and base saturation of the soil were not affected by the addition of $\mathrm{CaCO}_{3}$ solution. The application of $\mathrm{CaCO}_{3}$ microparticles in solution to the sowing furrow increased the common bean yield. Our results suggest that the application of $\mathrm{CaCO}_{3}$ microparticles to the sowing furrow was efficient to change soil chemical properties in the furrow soil and provided significant increases in the common bean yield.

Key words: Phaseolus vulgaris L., early development, soil correction, calcium carbonate, Cerrado.

\footnotetext{
Submetido em 09/09/2014 e aprovado em 08/10/2015.

${ }^{1}$ Embrapa Arroz e Feijão, Santo Antônio de Goiás, Goiás, Brasil. adriano.nascente @embrapa.br ${ }^{4}$ Embrapa Arroz e Feijão, Santo Antônio de Goiás, Goiás, Brasil. tarcisio.cobucci@embrapa.br Autor para correspondência: adriano.nascente@embrapa.br
} 


\section{INTRODUÇÃO}

A acidez do solo limita a produção agrícola em diversas áreas do mundo, em decorrência da toxidez causada por alumínio, pela baixa saturação por bases e pela menor disponibilidade de fósforo (P) (Fageria \& Baligar, 2008). No Brasil, o material mais utilizado como corretivo de acidez é o calcário, que é eficaz para elevar o pH, os teores de cálcio e de magnésio, a saturação por bases e para reduzir os teores de alumínio trocáveis no solo (Caires et al., 2005). Uma das formas de calcular a quantidade de calcário a ser aplicada no solo é o método que se baseia na elevação da saturação por bases a valores considerados adequados para uma dada cultura (Fageria et al., 2011; Pagani \& Mallarino, 2012).

$\mathrm{Na}$ maioria das áreas produtoras de grãos em sistema plantio direto (SPD), a correção da acidez do solo tem sido realizada mediante aplicação de calcário na superfície, sem incorporação (Soratto \& Crusciol, 2008; Alleoni et al., 2010). Entretanto, a reação do calcário é mais rápida no local de sua aplicação e incorporação, uma vez que o corretivo apresenta baixa mobilidade no solo e sua reação, dentre outros fatores, depende do seu contato com a solução do solo. Ou seja, para que ocorra a reação em profundidade é necessário que o calcário se mova para camadas mais profundas, o que acarreta demora na reação do corretivo no solo maior do que a da aplicação convencional com preparo do solo (Fageria \& Baligar, 2008; Soratto \& Crusciol, 2008; Caires et al., 2008). Resultados em campo mostram que o movimento do calcário em profundidade varia de acordo com o tempo e quantidade aplicada, tipo de solo, condições climáticas, utilização de fertilizantes ácidos e o sistema de cultivo (Caires et al., 2008; Churka Blum et al., 2013). De acordo com Caires et al. (2005), a calagem, com ou sem incorporação, proporciona maior controle da acidez do solo na camada de 0-5 cm, um ano após sua aplicação, e pode chegar a 10-20 $\mathrm{cm}$, após dois anos e meio. Entretanto, quando o calcário é incorporado, ocorre maior reação no solo entre 5-10 e 10-20 cm. Logo, essa demora para o calcário reagir com o solo em profundidade, por causa de sua baixa mobilidade, pode prejudicar o desenvolvimento das culturas agrícolas (Fageria \& Baligar, 2008).

Tecnologias que melhorem o ambiente radicular, para que ocorra o desenvolvimento das culturas, mais rapidamente, podem ser complemento da aplicação do calcário a lanço, sem incorporação. Portanto, a aplicação de solução de micropartículas de carbonato de cálcio no sulco de semeadura pode proporcionar a reação do calcário com o solo, de modo mais rápido, nesse sulco de semeadura, onde serão emitidas as raízes, aumentar-lhe o $\mathrm{pH}$ e a disponibilidade de fósforo no sulco de plantio, com consequentes incrementos na produtividade da cultura.
Enquanto o calcário convencional tem o tamanho das partículas variando de $50 \%<0,3 \mathrm{~mm}$ e $50 \%>0,3 \mathrm{~mm}$, as micropartículas têm entre 0,0001 e 0,0002 mm. Assim, quando se usa o calcário aplicado na superfície do solo, sem incorporação, considerando-se 1 ha, tem-se a intenção de corrigir todo o solo de uma superfície de $10000 \mathrm{~m}^{2}$ até $20 \mathrm{~cm}$ de profundidade. Por outro lado, quando se utiliza calcário na forma de micropartículas, tem-se a intenção de influenciar somente o solo no sulco de semeadura, o que pode melhorar as condições para o desenvolvimento inicial da cultura.

Considerando-se o espaçamento utilizado para o feijoeiro-comum, com o uso da correção à base de calcário em micropartículas localizadas no sulco, corrige-se, em 1 ha, o volume de solo localizado sob 1.000 $\mathrm{m}^{2}$ de área $(20.000 \mathrm{~m}$ de sulcos x 0,05m), ou seja, dez vezes menos do que quando se aplica o calcário pelo método convencional. Por causa do tamanho reduzido das micropartículas de calcário, é provável que a aplicação desse tipo de corretivo possa proporcionar a reação mais rápida e localizada no sulco de semeadura, em relação à aplicação tradicional. Porém, não foram encontrados estudos que avaliassem essa prática. Por esta razão, o objetivo deste trabalho foi o de testar a hipótese de que a aplicação localizada de solução de micropartículas de calcário $\left(\mathrm{CaCO}_{3}\right)$ no sulco de semeadura pode acelerar o contato do corretivo com o solo, em torno das raízes, e sua reação no solo, alterando momentaneamente os atributos químicos do solo e aumentando a produtividade do feijoeiro-comum.

\section{MATERIAL E MÉTODOS}

\section{Caracterização dos ambientes}

Os experimentos foram conduzidos em dois locais:

1. Fazenda Guaribas, no município de Unaí, MG. Neste local, foram conduzidos os ensaios do experimento 1 , em 2009 e 2010, e os ensaios do experimento 2, em 2010 e 2011. O local encontra-se nas coordenadas $16^{\circ}$ $21^{\prime} 27^{\prime \prime} \mathrm{S}$ e $46^{\circ} 54^{\prime} 22^{\prime \prime} \mathrm{O}$, em altitude de $575 \mathrm{~m}$. A precipitação média anual do município é de aproximadamente $1.200 \mathrm{~mm}$. Segundo a classificação de Köppen, o clima é do tipo Aw, tropical de savana, megatérmico, com temperatura média anual do ar de $27^{\circ} \mathrm{C}$. Durante a condução dos experimentos (maio-agosto), as médias das precipitações dos dois anos foram: maio, $22 \mathrm{~mm}$; junho, $7 \mathrm{~mm}$; julho, $10 \mathrm{~mm}$; e agosto, $12 \mathrm{~mm}$.

2. Fazenda Chimarrão, em Paracatu, MG. Neste local, foram conduzidos os ensaios do experimento 2, em 2010 e em 2011. O local encontra-se nas coordenadas $17^{\circ}$ 13' 19" S e 46 52' 30" O, em altitude de $688 \mathrm{~m}$. A precipitação média anual é de aproximadamente $1.438 \mathrm{~mm}$. 
Segundo a classificação de Köppen, o clima é do tipo Aw, tropical de savana, megatérmico, com temperatura média anual do ar de $24,4{ }^{\circ} \mathrm{C}$. Durante a condução dos experimentos (maio-agosto), as médias das precipitações dos dois anos foram: maio, $25 \mathrm{~mm}$; junho, $3 \mathrm{~mm}$; julho, $8 \mathrm{~mm}$; e agosto, $9 \mathrm{~mm}$.

As áreas experimentais, localizadas sob pivô-central, haviam sido cultivadas por três (experimento 1 ), cinco (experimento 2, Unaí) e quatro (experimento 2, Paracatu) anos no sistema plantio direto (SPD), com milho no verão e feijão no inverno. Os solos foram classificados como Latossolo Vermelho- Escuro com textura argilosa. No primeiro ano de condução, antes da instalação dos experimentos, foram feitas amostragens para a caracterização química (Tabela 1) e a análise de solo foi realizada de acordo com os métodos descritos em Embrapa (1997).

\section{Experimento 1}

Esse experimento, conduzido na Fazenda Guaribas, foi repetido nas safras de inverno de 2009 e 2010. Nele se estudaram doses de $\mathrm{CaCO}_{3}$ - $\mathrm{O}$ delineamento experimental foi em blocos ao acaso, com quatro repetições. Os tratamentos constituíram-se de seis doses de solução, contendo $\mathrm{CaCO}_{3}^{-}(0,1.050,2.100,4.200,8.400$ e 12.600 $\mathrm{g} \mathrm{ha}^{-1}$ ), aplicadas no sulco, no dia da semeadura da cultura
A necessidade de calcário, calculada para elevar a saturação por bases para $60 \%$, resultou na dose de 7,1 $\mathrm{kg} \mathrm{ha}^{-1}$. Considerando-se que o volume de solo a ser corrigido com a utilização de micropartículas de calcário no sulco de semeadura é dez vezes menor do que o corrigido, quando o calcário é aplicado em área total, a quantidade de calcário que deveria ser aplicada no sulco seria de $710 \mathrm{~g} \mathrm{ha}^{-1}$. Entretanto, com os produtos disponíveis no mercado, não foi possível estabelecer doses menores que $1.050 \mathrm{~g} \mathrm{ha}^{-1}$, que foram usados como limite inferior das doses crescentes, empregadas neste estudo, e que variaram entre 0 e $12.600 \mathrm{~g} \mathrm{ha}^{-1}$.

\section{Experimento 2}

Nesse experimento, conduzido nas safras 2010 e 2011, avaliou-se a dose de $1.050 \mathrm{~g} \mathrm{ha}^{-1} \mathrm{de} \mathrm{CaCO}_{3}$, na Fazenda Guaribas e na Fazenda Chimarrão. Foi escolhida a dose de $1.050 \mathrm{~g} \mathrm{ha}^{-1}$, menor dose possível, considerando-se os produtos disponíveis no mercado, dado o fato da necessidade de a calagem, calculada a partir dos dados de análise do solo e ajustada conforme o descrito anteriormente, resultar em doses baixas, menores que esse valor. $\mathrm{O}$ delineamento experimental foi em blocos ao acaso, com oito repetições, num esquema fatorial 2 (com ou sem solução com $\mathrm{CaCO}_{3}$ ) x 4 (ambientes). Os tratamentos foram a ausência e presença de solução com

Tabela 1: Características químicas iniciais dos solos das áreas onde foram conduzidos os experimentos na profundidade de 0-20 cm

\begin{tabular}{|c|c|c|c|c|c|c|c|c|c|}
\hline \multicolumn{10}{|c|}{ Unaí - MG 2009 (experimento 1) } \\
\hline \multirow[b]{2}{*}{$(\mathrm{cm})$} & \multirow{2}{*}{$\begin{array}{c}\text { pH } \\
\text { (água) }\end{array}$} & \multirow{2}{*}{$\begin{array}{l}\text { M.O. } \\
\mathrm{g} \mathrm{dm}^{-3}\end{array}$} & & $\mathbf{C a}$ & Mg & Al & $\mathrm{H}+\mathrm{Al}$ & $\mathbf{V}$ & $\mathbf{m}$ \\
\hline & & & \multicolumn{4}{|c|}{$\mathrm{mmol}_{\mathrm{c}} \mathrm{dm}^{-3}$} & & \multicolumn{2}{|c|}{$\%$} \\
\hline $0-20$ & 5,0 & 1,5 & \multicolumn{2}{|r|}{2,4} & 0,9 & 0,3 & 2,6 & 59,1 & 3 \\
\hline $\mathbf{K}$ & $\mathbf{P}$ & B & $\mathbf{C u}$ & $\mathbf{F e}$ & Mn & $\mathbf{Z n}$ & Argila & Silte & Areia \\
\hline \multicolumn{2}{|c|}{$\mathbf{m g ~ d m}{ }^{-3}$} & \multicolumn{5}{|c|}{$\mathbf{m g ~ d m}{ }^{-3}$} & \multicolumn{3}{|c|}{$\mathrm{g} \mathrm{kg}^{-1}$} \\
\hline$\overline{19,8}$ & 12,1 & 0,8 & 1,2 & 17,3 & 21,5 & 9,7 & 500 & 300 & 200 \\
\hline \multicolumn{10}{|c|}{ Unaí - MG 2010 (experimento 2) } \\
\hline & pH & M.O. & & $\mathbf{C a}$ & Mg & Al & $\mathbf{H}+\mathbf{A l}$ & $\mathbf{V}$ & $\mathbf{m}$ \\
\hline$(\mathbf{c m})$ & (água) & $\mathbf{g ~ d m ^ { - 3 }}$ & \multicolumn{4}{|c|}{$\mathbf{m m o l}_{\mathrm{c}} \mathbf{d m}^{-3}$} & & \multicolumn{2}{|c|}{$\%$} \\
\hline $0-20$ & 5,6 & 3,6 & & 2,5 & 1 & 0,1 & 5,0 & 44 & 2 \\
\hline $\mathbf{K}$ & $\mathbf{P}$ & B & $\mathbf{C u}$ & $\mathbf{F e}$ & Mn & $\mathbf{Z n}$ & Argila & Silte & Areia \\
\hline \multicolumn{2}{|c|}{$\mathrm{mg} \mathrm{dm}^{-3}$} & \multicolumn{5}{|c|}{$\mathrm{mg} \mathrm{dm}^{-3}$} & \multicolumn{3}{|c|}{$\mathrm{g} \mathrm{kg}^{-1}$} \\
\hline 19,4 & 16,8 & 1 & 1,4 & 21,2 & 25,3 & 10,3 & 510 & 310 & 180 \\
\hline \multicolumn{10}{|c|}{ Paracatu - MG 2010 (experimento 2) } \\
\hline & pH & M.O. & & $\mathbf{C a}$ & Mg & Al & $\mathbf{H}+\mathbf{A l}$ & $\mathbf{V}$ & $\mathbf{m}$ \\
\hline (cm) & (água) & $\mathrm{g} \mathrm{dm}^{-3}$ & \multicolumn{4}{|c|}{$\mathrm{mmol}_{\mathrm{c}} \mathrm{dm}^{-3}$} & & \multicolumn{2}{|c|}{$\%$} \\
\hline $0-20$ & 5,5 & 1,9 & \multicolumn{2}{|r|}{4,1} & 2,1 & 0,0 & 5,0 & 71,6 & 0 \\
\hline $\mathbf{K}$ & $\mathbf{P}$ & B & $\mathrm{Cu}$ & $\mathrm{Fe}$ & Mn & $\mathbf{Z n}$ & Argila & Silte & Areia \\
\hline \multicolumn{2}{|c|}{$\mathrm{mg} \mathrm{dm} \mathbf{m}^{-3}$} & \multicolumn{5}{|c|}{$\mathrm{mg} \mathrm{dm}^{-3}$} & \multicolumn{3}{|c|}{$\mathrm{g} \mathrm{kg}^{-1}$} \\
\hline 22,0 & 26,8 & 0,8 & 2,6 & 18 & 44,5 & 11,5 & 652 & 193 & 180 \\
\hline
\end{tabular}

Extrator: P-Mehlich 1; B-Água quente; $\mathrm{Cu} / \mathrm{Fe} / \mathrm{Mn} / \mathrm{Zn}$ - DTPA. 
$\mathrm{CaCO}_{3}$, na dose de $1.050 \mathrm{~g} \mathrm{ha}^{-1}$, aplicados no sulco no dia da semeadura. As parcelas tinham 4,0 x $10 \mathrm{~m}$ e a área útil constou das duas linhas centrais, desprezando-se 0,50 $\mathrm{m}$ de cada extremidade das fileiras.

\section{Implantação da cultura}

A semeadura do feijoeiro-comum, cultivar Pérola, foi realizada mecanicamente na primeira quinzena de maio, nos dois experimentos, no espaçamento de 0,50 $\mathrm{m}$ entrelinhas e densidade de oito sementes por metro. A adubação no sulco de semeadura foi de $30 \mathrm{~kg} \mathrm{ha}^{-1}$ de $\mathrm{N}$ (ureia); 39,3 $\mathrm{kg} \mathrm{ha}^{-1}$ de P $+10 \mathrm{~kg} \mathrm{ha}^{-1}$ de $\mathrm{N}$ (MAP, monoamônio fosfato) e de $50 \mathrm{~kg} \mathrm{ha}^{-1}$ de $\mathrm{K}$ (cloreto de potássio). Em cobertura, usaram-se $60 \mathrm{~kg} \mathrm{ha}^{-1} \mathrm{de} \mathrm{N}$ (ureia), aos 18 DAE (dias após a emergência). O manejo fitossanitário da cultura foi realizado de acordo com as necessidades (Vieira et al., 2006). Na média, a emergência das plantas ocorreu aos sete dias após a semeadura e, o florescimento, aos 33 dias após a emergência (DAE). A colheita foi efetuada manualmente, após a maturação fisiológica da cultura. O feijoeiro-comum apresentou média de ciclo de 103 DAE.

Quanto à irrigação, utilizou-se o sistema por aspersão convencional. No manejo de água, foram utilizados três coeficientes de cultura (Kc), distribuídos em quatro períodos compreendidos entre a emergência e a colheita. Para a fase vegetativa, foi utilizado o valor de 0,4; para a fase reprodutiva foram dois os valores de $\mathrm{Kc}$, o inicial de 0,7 e o final de $1,0 \mathrm{e}$, para a fase de maturação, esses valores foram invertidos, ou seja, o inicial de 1,0 e o final de 0,7. Assim, o controle da irrigação, considerando-se a profundidade de exploração do sistema radicular, de $0,2 \mathrm{~m}$, foi iniciado com a capacidade de água disponível (CAD) no seu máximo, subtraindo-se, sucessivamente, o valor da evapotranspiração da cultura, até que o total de água atingisse limite mínimo de 40\% da CAD (Doorenbos \& Pruitt, 1976).

A solução de calcário foi adquirida de empresa privada. Essa solução havia sido preparada pela mistura de partículas finas (entre 0,0001 e 0,0002 mm) de calcário com água. A aplicação da solução de $\mathrm{CaCO}_{3}$ foi feita diretamente no sulco de plantio, com aplicador StandMax Hunter CS, acoplado à semeadora. O aplicador possui sistema automático de comando por sensor indutivo, que liga e desliga a injeção quando a semeadora é levantada ou baixada. Utilizou-se volume de calda de $60 \mathrm{~L} \mathrm{ha}^{-1}$. Na dose zero, aplicou-se apenas água. A composição química das partículas de calcário é a mesma do calcário tradicional, tendo como única diferença o tamanho das partículas. O equipamento de aplicação do calcário líquido tem dispositivo para manter a solução em suspensão durante o período de aplicação.

\section{Avaliações}

No experimento 1 , foram realizadas amostragens do solo aos 15 e 30 dias após a aplicação da solução com $\mathrm{CaCO}_{3}$, na camada de $0-0,20 \mathrm{~m}$ de profundidade, na linha e na entrelinha $(0,25 \mathrm{~cm}$ da linha). Foram retiradas, aleatoriamente, com trado de rosca, oito amostras simples, por parcela, para constituir uma amostra composta. As amostras compostas foram secadas ao ar e peneiradas (malha $2 \mathrm{~mm}$ ). Posteriormente, elas foram analisadas quanto a $\mathrm{pH}\left(\mathrm{CaCl}_{2} 0,01 \mathrm{~mol} \mathrm{~L}^{-1}\right), \mathrm{P}, \mathrm{Al}, \mathrm{Ca}$ e $\mathrm{K}$ trocáveis. Também foi calculada a saturação por bases (V\%), conforme metodologia proposta por Embrapa (1997).

Nos dois experimentos, foram determinadas as seguintes variáveis: a) número de vagens por planta: contagem do número de vagens de dez plantas, coletada, ao acaso, em cada unidade experimental, durante a colheita; b) número de grãos por vagem: contagem do número de grãos das dez plantas, coletada, ao acaso, durante a colheita; c) massa de 100 grãos: determinada pela coleta, ao acaso, e pesagem de quatro amostras de 100 grãos de cada unidade experimental, com posterior correção do teor de água dos grãos para $130 \mathrm{~g} \mathrm{~kg}^{-1}$; d) produtividade de grãos: determinada após o arranquio manual das plantas da área útil de cada unidade experimental, trilha mecânica e pesagem dos grãos. Foi determinado o peso dos grãos colhidos e calculada a produtividade de grãos $\left(\mathrm{kg} \mathrm{ha}^{-1}\right)$, após o teor de água ser corrigido para $130 \mathrm{~g} \mathrm{~kg}^{-1}$.

\section{Análise estatística}

Os dados obtidos no experimento 1 , doses de $\mathrm{CaCO}_{3}$, foram submetidos à análise de variância e regressão, considerando-se doses, anos e interação doses x anos como fontes de variação. No experimento 2 , os dados foram submetidos à análise de variância, considerandose blocos, ambientes e interação blocos $\mathrm{x}$ ambientes como causas da variação. Utilizou-se o pacote estatístico SAS.

\section{RESULTADOS E DISCUSSÃO}

\section{Experimento 1 (doses de $\mathrm{CaCO}_{3}$ )}

Não houve interação entre os fatores anos e doses de calcário, em todas as variáveis (Tabela 2). Logo, apresentaram-se os dados da média dos anos (Figuras 1 a 4). $\mathrm{Na}$ entrelinha do feijoeiro $(0,25 \mathrm{~m}$ da linha), não foram verificados efeitos da aplicação de $\mathrm{CaCO}_{3}$ na concentração dos nutrientes no solo. Assim, esses resultados podem ser indicativos de que a aplicação do corretivo tem ação pontual, próxima à área de aplicação, e não afeta a área total do solo.

$\mathrm{Na}$ linha de semeadura, os teores de $\mathrm{Ca}$, K e a saturação de bases no solo não foram influenciados pela adi- 
ção da solução com $\mathrm{CaCO}_{3}$. Com relação ao $\mathrm{pH}$ do solo no sulco de plantio, houve efeito significativo da aplicação da solução com micropartículas de calcário, nas duas épocas (Tabela 2); os dados foram ajustados a equações lineares (Figura 1). Segundo Caires et al. (2008), um dos efeitos do calcário é aumentar o $\mathrm{pH}$ do solo. Os valores de $\mathrm{pH}$ obtidos aos 30 dias foram numericamente superiores aos obtidos aos 15 dias, o que pode indicar que o calcário reagiu com o solo entre 15 e 30 dias. O efeito do calcário aplicado de forma tradicional varia de acordo com o tempo e a quantidade aplicada, o tipo de solo, as condições climáticas, a utilização de fertilizantes ácidos e o sistema de cultivo (Caires et al., 2008; Churka Blum et al., 2013); logo, espera-se que a aplicação de solução de calcário no sulco de semeadura também seja influenciada por esses fatores.

A aplicação do corretivo no sulco também proporcionou alteração significativa dos teores de $\mathrm{P}$ no solo, na linha de semeadura, nas duas épocas (Tabela 2), com ajuste dos dados a equações quadráticas (Figura 2). Em relação à saturação por $\mathrm{Al}^{+3}$ do solo, os dados foram ajustados a equação linear, com valores decrescentes à medida que se aumentaram os teores de $\mathrm{CaCO}_{3}$ aplicados (Figura 3).
Houve maior disponibilidade de P no sulco de semeadura, nas duas épocas, por causa da aplicação da solução com $\mathrm{CaCO}_{3}$ no sulco de semeadura. Essa maior disponibilidade de $\mathrm{P}$ pode ter ocorrido por liberação do P fixado ao Al ou Fe. Bair \& Davenport (2012) também verificaram aumento da disponibilidade de fósforo com o aumento do pH. Segundo Souza et al. (2006), os solos tropicais são altamente intemperizados e neles predominam os minerais de argila 1:1, como a caulinita e os óxidos de Fe (hematita e goethita) e Al (gibbsita), que apresentam alta capacidade de adsorção de P. Souza et al. (2010) acrescentam que o processo de adsorção de $\mathrm{P}$ pelos óxidos, hidróxidos e oxiidróxidos de ferro e alumínio é um dos principais fatores envolvidos na insolubilização desse nutriente, em solos tropicais. Assim, é provável que a aplicação de solução de calcário no sulco de semeadura tenha proporcionado maior disponibilidade de $\mathrm{P}$, no início do desenvolvimento da cultura. A elevação do $\mathrm{pH}$ do solo pela calagem aumenta a concentração e a atividade dos íons $\mathrm{OH}^{-}$em solução, aumenta a precipitação de $\mathrm{Fe}$ e a de $\mathrm{Al}$, reduz a precipitação de P-Fe e P-Al (Mendez \& Kamprath, 1978; Alleoni et al., 2010).

Tabela 2: Análise de variância dos teores de nutrientes no solo da linha de cultivo, na profundidade de 0-20 cm, aos 15 e 30 dias após a semeadura do feijoeiro-comum, componentes de produção e produtividade da cultura, em função das doses de $\mathrm{CaCO}_{3}$ aplicados no sulco de semeadura (experimento 1)

\begin{tabular}{|c|c|c|c|c|c|c|}
\hline \multirow{3}{*}{ Fatores } & \multicolumn{6}{|c|}{15 dias após a semeadura } \\
\hline & \multicolumn{6}{|c|}{ Variáveis } \\
\hline & pH & $\mathbf{P}$ & $\mathbf{K}$ & $\mathbf{C a}$ & Al & $\mathbf{V}$ \\
\hline & \multicolumn{6}{|c|}{ Probabilidade do teste $\mathbf{F}$} \\
\hline Blocos & 0,084 & 0,507 & 0,389 & 0,375 & 0,064 & 0,205 \\
\hline Ano (A) & 0,171 & 0,210 & 0,462 & 0,236 & 0,376 & 0,387 \\
\hline Tratamento $(\mathrm{T})$ & 0,002 & 0,001 & 0,824 & 0,052 & 0,005 & 0,284 \\
\hline \multirow[t]{2}{*}{$\mathrm{A} \times \mathrm{T}$} & 0,264 & 0,387 & 0,997 & 0,201 & 0,148 & 0,775 \\
\hline & \multicolumn{6}{|c|}{30 dias após a semeadura } \\
\hline \multirow[t]{3}{*}{ Fatores } & \multicolumn{6}{|c|}{ Variáveis } \\
\hline & pH & $\mathbf{P}$ & $\mathbf{K}$ & $\mathbf{C a}$ & Al & $\mathbf{V}$ \\
\hline & \multicolumn{6}{|c|}{ Probabilidade do teste $\mathbf{F}$} \\
\hline Blocos & 0,329 & 0,838 & 0,059 & 0,736 & 0,363 & 0,082 \\
\hline Ano (A) & 0,225 & 0,178 & 0,112 & 0,229 & 0,119 & 0,071 \\
\hline Tratamento $(\mathrm{T})$ & 0,001 & 0,001 & 0,388 & 0,847 & 0,003 & 0,437 \\
\hline \multirow[t]{2}{*}{$\mathrm{A} \times \mathrm{T}$} & 0,051 & 0,051 & 0,739 & 0,997 & 0,578 & 0,773 \\
\hline & \multicolumn{6}{|c|}{ Variáveis } \\
\hline \multirow[t]{2}{*}{ Fatores } & \multicolumn{2}{|c|}{ N. vagens } & Grãos vagens $^{-1}$ & \multicolumn{2}{|c|}{ Massa de 100 sementes } & Produtividade \\
\hline & \multicolumn{6}{|c|}{ Probabilidade do teste $\mathbf{F}$} \\
\hline Blocos & & & 0,359 & & 0,999 & 0,999 \\
\hline Ano (A) & & & 0,247 & & 0,245 & 0,114 \\
\hline Tratamento $(\mathrm{T})$ & & & 0,999 & & 0,999 & 0,001 \\
\hline $\mathrm{A} \times \mathrm{T}$ & & & 0,846 & & 0,987 & 0,131 \\
\hline
\end{tabular}



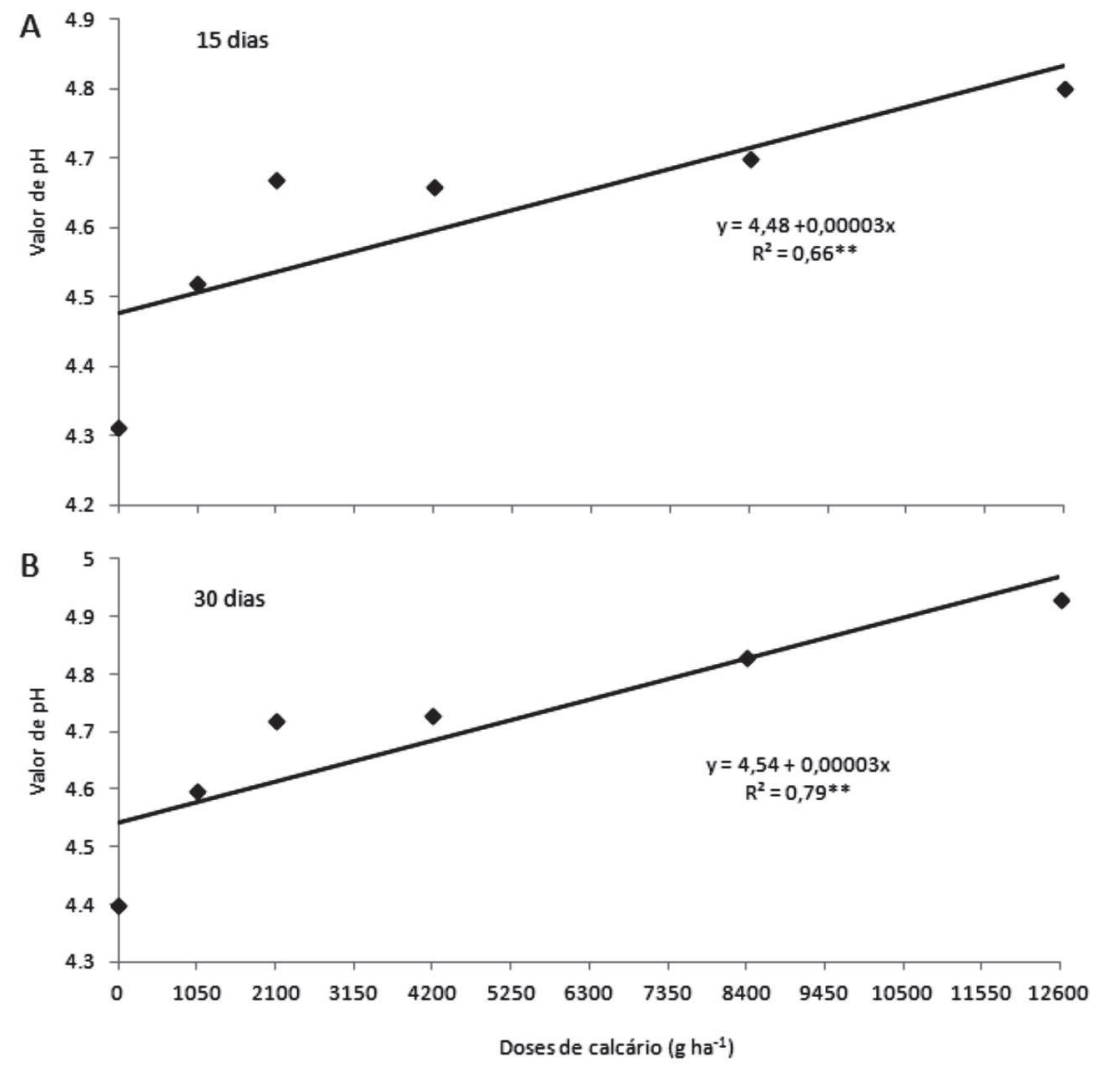

Figura 1: Valores de $\mathrm{pH}$ do solo, na profundidade de $0-20 \mathrm{~cm}$, em função das doses de $\mathrm{CaCO}_{3}$ aplicadas no sulco de semeadura do feijoeiro-comum, aos 15 (A) e 30 (B) dias após a semeadura da cultura. Média de duas safras agrícolas.
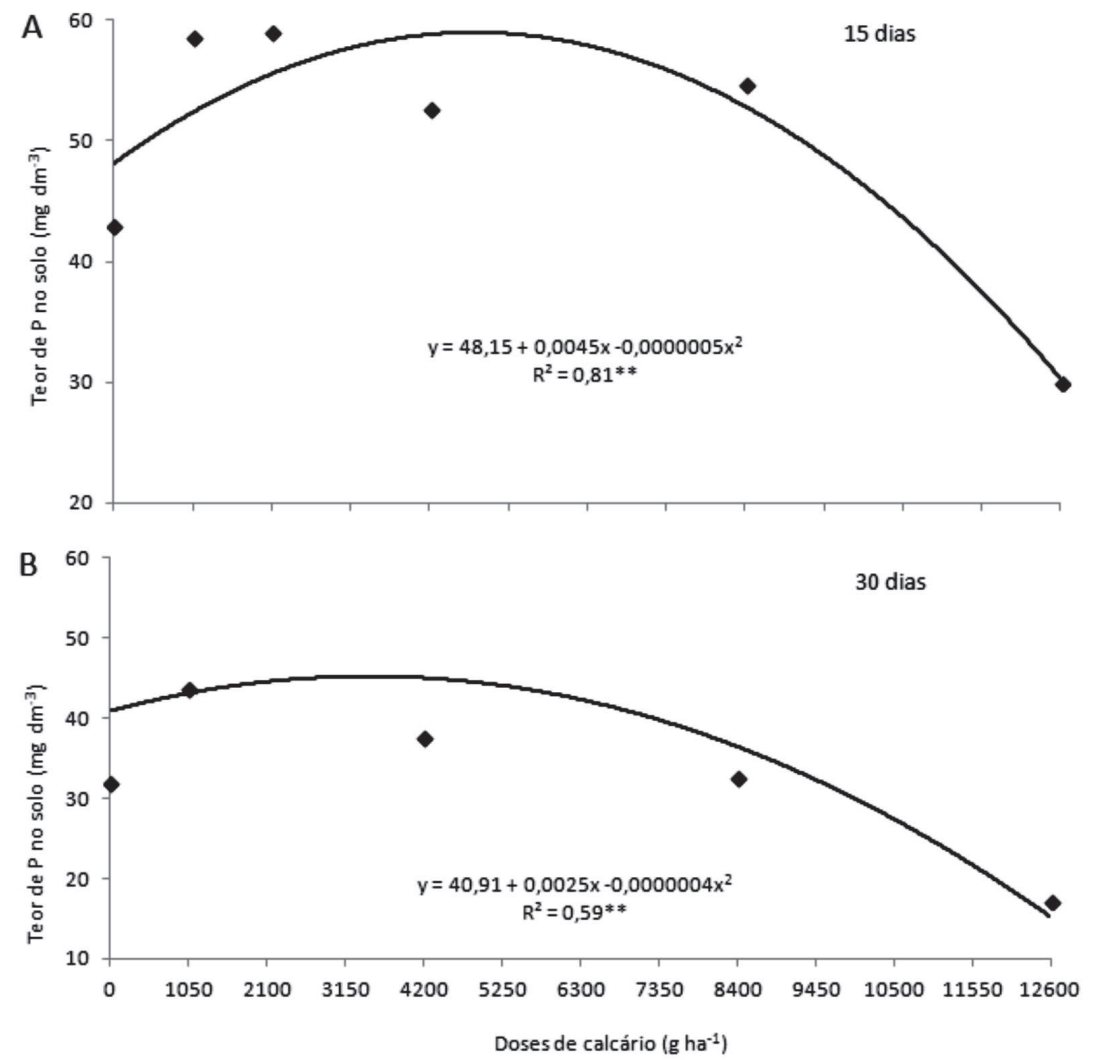

Figura 2: Teores de fósforo do solo, na profundidade de 0-20 cm, em função das doses de $\mathrm{CaCO}_{3}$ aplicadas no sulco de semeadura do feijoeiro-comum aos 15 (A) e 30 (B) dias após a semeadura da cultura. Média de duas safras agrícolas. 
Entretanto, vale ressaltar que a aplicação de calcário líquido aumentou os teores de $\mathrm{P}$ no solo até determinado ponto (4.700 g de $\mathrm{CaCO}_{3}$, aos 15 dias, e $2.900 \mathrm{~g}$ de $\mathrm{CaCO}_{3}$, aos 30 dias) (Figura 2). A partir desses pontos os teores de $\mathrm{P}$ no solo decresceram. A calagem, ao alterar o $\mathrm{pH}$ do solo, neutraliza as cargas positivas na superfície de argilas não silicatadas de óxidos e hidróxidos de Fe e Al, podendo revertê-las em cargas residuais negativas. Isso "bloqueia" sítios de adsorção de $\mathrm{P}$ no solo e pode reduzir a "adsorção específica de P". Também é conhecido o fenômeno de fixação de $\mathrm{P}$ de forma temporária, em função da formação de hidróxidos de $\mathrm{Al}$ amorfos intermediários $\left[\mathrm{Al}(\mathrm{OH})^{2+}, \mathrm{Al}(\mathrm{OH})_{2}^{+}\right]$, muito reativos, quando da aplicação de calcário. Esse fenômeno é temporário, pois é revertido com o avanço da reação do calcário no solo, e a fixação de $\mathrm{P}$ pelo Al fica novamente insignificante, quando a elevação do $\mathrm{pH}$ proporciona predominância de formas precipitadas de $\mathrm{Al}(\mathrm{OH})_{3}$ (Fageria \& Baligar, 2008; Souza et al., 2010; Fageria et al., 2011). Esse fenômeno pode ser uma das explicações para a redução do P com o aumento da dose de solução de calcário no sulco. Outra possível explicação seria a abundância de cálcio, que normalmente ocorre em $\mathrm{pH}$ alto, o que pode formar compostos de $\mathrm{P}$ e precipitá-lo, tornando-o indisponível para as plantas (Malavolta et al., 1997).
No entanto, os resultados apresentados permitem inferir que a aplicação localizada de micropartículas de calcário em solução pode reagir mais rápido, no sulco de semeadura que na aplicação convencional, e proporcionar maior disponibilidade de $\mathrm{P}$ no sulco de plantio, nos estádios iniciais de desenvolvimento da planta. Os valores de $\mathrm{pH}, \mathrm{Pe} \mathrm{Al}$, observados na avaliação aos 30 dias, indicam que o calcário ainda estava reagindo no solo, em comparação com os valores obtidos aos 15 dias, o que pode sugerir que as reações vão além de 30 dias. Portanto, estudos futuros poderiam ser realizados para verificar por quanto tempo essas pequenas quantidades de calcário líquido aplicadas no sulco de semeadura, alteram essas características do solo. Além disso, a aplicação de calcário líquido, aliada à calagem tradicional, pode proporcionar resultados mais promissores do que somente a calagem tradicional. Esta, no sistema plantio direto, demora ainda mais a reagir com o solo (Caires et al., 2008; Briedis et al., 2012).

Com relação aos componentes de produção e produtividade, verificaram-se efeitos significativos das doses de $\mathrm{CaCO}_{3}$ no número de vagens por planta e na produtividade (Tabela 2), sendo os dados ajustados à equações quadráticas (Figura 4). Esse aumento de produtividade pode ter ocorrido por aumento da disponibilidade de $\mathrm{P}$

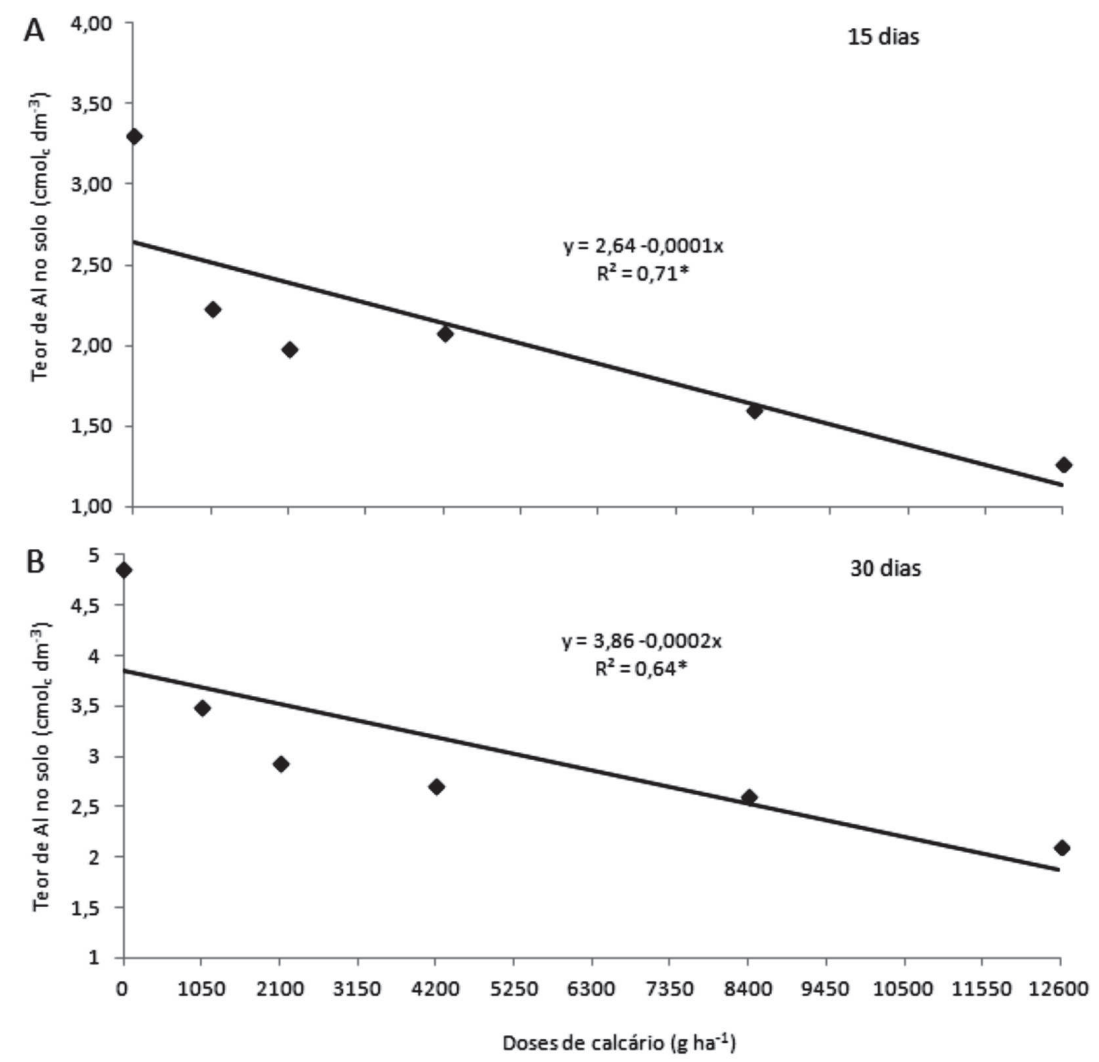

Figura 3: Teores de alumínio do solo, na profundidade de 0-20 cm, em função das doses de $\mathrm{CaCO}_{3}$ aplicadas no sulco de semeadura do feijoeiro-comum aos 15 (A) e 30 (B) dias após a semeadura da cultura. Média de duas safras agrícolas. 
no sulco de semeadura, que afeta significativamente essa variável no feijoeiro-comum (Miranda et al., 2005; Pelá et al., 2009; Silva et al., 2001; Valderrama et al., 2009; Zucareli et al., 2011).

Outros fatores, além da disponibilidade de fósforo, contribuíram para o aumento da produtividade de grãos do feijoeiro-comum, uma vez que a dose da solução com calcário que proporcionou a maior produtividade da cultura foi bem maior do que a dose que acarretou os maiores teores de $\mathrm{P}$ no solo. Esses fatores podem ser a redução da solubilidade do Al, ou o aumento da disponibilidade de $\mathrm{Ca}, \mathrm{Mg}$ e $\mathrm{K}$ no solo, ou outros fatores que não foram avaliados neste estudo.

Os resultados sugerem que a aplicação de pequena quantidade de solução com calcário na forma de micropartículas aumentou a disponibilidade de $\mathrm{P}$, até determinado ponto, elevou o $\mathrm{pH}$ do solo e reduziu a saturação de Al no sulco de semeadura e aumentou a produtividade de grãos. Entretanto, o aumento dos teores de nutrientes foi limitado ao sulco de semeadura, uma vez que não houve efeito nas entrelinhas da cultura. Portanto, não deve ser prática utilizada visando à correção total do solo, ao aumento da saturação de bases e ao fornecimento de Ca para a cultura. Necessário se faz desenvolver estudos para avaliar o efeito combinado das calagens, no sulco e em superfície, na fertilidade do solo, no longo prazo.
Além disso, com base nos resultados obtidos, verifica-se que mesmo em solos já corrigidos e com altos valores de saturação por bases (Tabela 1), ocorreram incrementos nos teores de $\mathrm{P}$ no solo e na produtividade da cultura. Estudos devem ser desenvolvidos para se verificar até quais valores de saturação por bases e de pH justifica-se a aplicação de calcário na linha de semeadura.

\section{Experimento 2. (Efeito da aplicação da dose de $1.050 \mathrm{~g} \mathrm{ha}^{-1}$ de $\mathrm{CaCO}_{3}$ em 4 ambientes)}

Quando se avaliou a menor dose de calcário (1.050 g $\mathrm{ha}^{-1}$ ), visando a reduzir custos para o produtor e verificar se causava efeito no feijoeiro, constatou-se que não houve interação entre a aplicação de calcário e os locais (Tabela 3). Por isso, apresentaram-se as médias dos locais. $\mathrm{O}$ efeito do calcário foi significativo no número de vagens $\mathrm{m}^{-2}$ e na produtividade de grãos. $\mathrm{O}$ maior número de vagens $\mathrm{m}^{-2}$ pode explicar a maior produtividade de grãos, obtida no tratamento com a utilização da solução com calcário, uma vez que esse componente de produção é o que mais afeta a produtividade de grãos na cultura do feijoeiro-comum (Fageria et al., 2011; Zucareli et al., 2011).

Por ser um trabalho inicial, muitas indagações ainda precisam ser elucidadas, como o critério para o agricul-
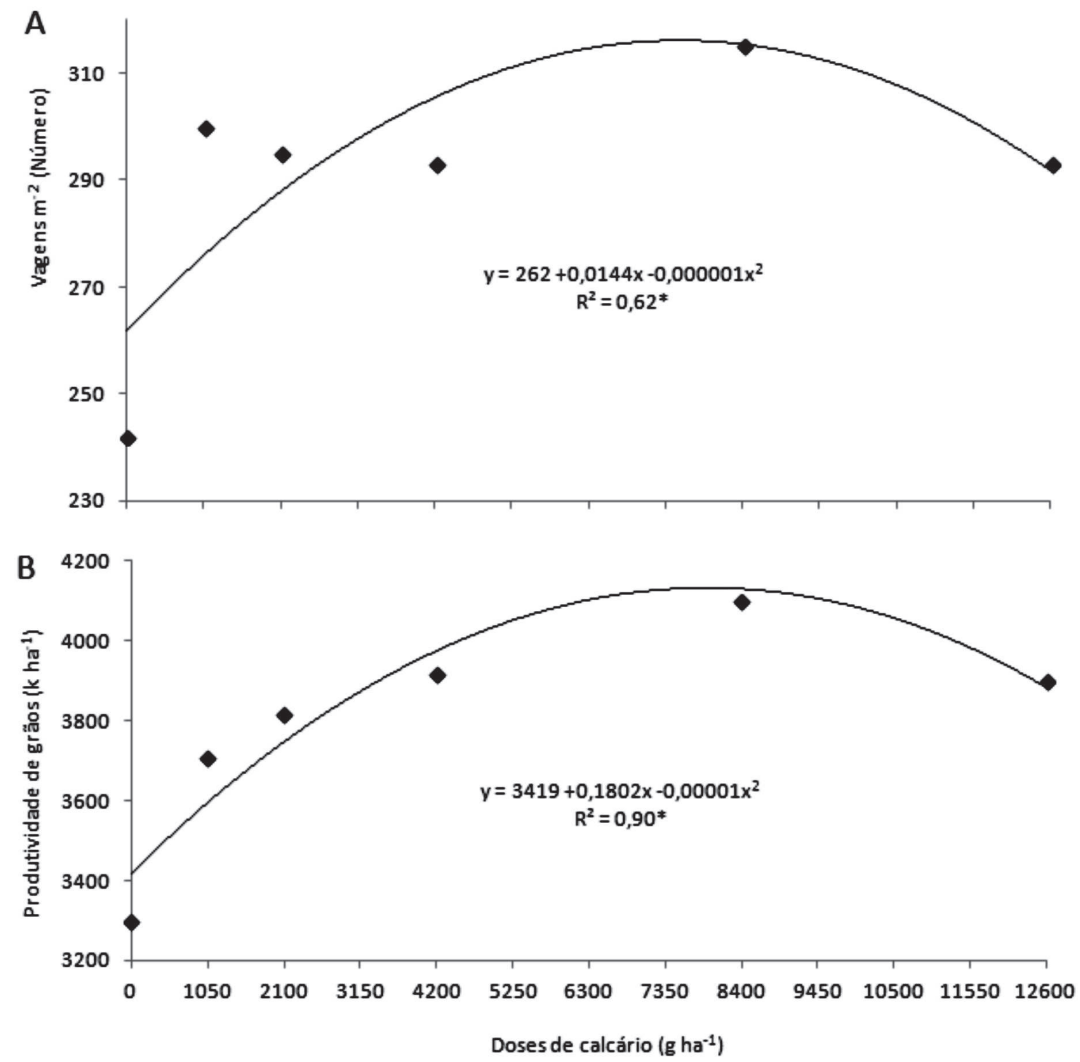

Figura 4: Número de vagens $\mathrm{m}^{-2}$ (A) e produtividade de grãos (B) do feijoeiro-comum, em função das doses de $\mathrm{CaCO}_{3}$ aplicadas no sulco de semeadura, média de duas safras agrícolas. 
Tabela 3: Componentes de produção e produtividade de grãos do feijoeiro-comum, afetados pela ausência e presença de $\mathrm{CaCO}_{3}$ (1.050 $\left.\mathrm{g} \mathrm{ha}^{-1}\right)$ e análise de variância (experimento 2)

\begin{tabular}{|c|c|c|c|c|c|}
\hline \multirow{2}{*}{ Tratamentos } & Unaí 2010 & Unaí 2011 & Paracatu 2010 & Paracatu 2011 & Média \\
\hline & \multicolumn{4}{|c|}{ Número vagens $\mathbf{m}^{-2}$} & \\
\hline 1 - Testemunha & 259 & 241 & 289 & 241 & 258 \\
\hline $2-\mathrm{CaCO}_{3}$ & 276 & 274 & 307 & 283 & $283^{*}$ \\
\hline \multicolumn{6}{|c|}{ Número grãos vagens ${ }^{-1}$} \\
\hline 1 - Testemunha & 4,5 & 4,9 & 4,8 & 4,9 & 4,8 \\
\hline $2-\mathrm{CaCO}_{3}$ & 5,0 & 4,8 & 5,0 & 4,8 & 4,9 \\
\hline \multicolumn{6}{|c|}{ Massa de 100 grãos } \\
\hline $1-$ Testemunha & 25,0 & 24,5 & 25,0 & 24,5 & 25,2 \\
\hline $2-\mathrm{CaCO}_{3}$ & 26,0 & 25,9 & 26,5 & 25,9 & 26,5 \\
\hline \multicolumn{6}{|c|}{ Produtividade kg ha' } \\
\hline $1-$ Testemunha & 2948 & 2943 & 3522 & 2937 & 3087 \\
\hline $2-\mathrm{CaCO}_{3}$ & 3600 & 3373 & 4080 & 3447 & $3624^{*}$ \\
\hline
\end{tabular}

Médias seguidas por *, diferem significativamente $(\mathrm{p} \leq 0,05)$ da testemunha.

tor escolher a dose a ser aplicada com base na saturação por bases, uma vez que em Paracatu e Unaí foram de 72 e de $44 \%$, respectivamente. O objetivo do trabalho não foi de elevar a saturação por bases nem de fazer a correção da área total, mas de melhorar as características químicas do sulco de semeadura, para proporcionar melhor desenvolvimento das plantas do feijoeiro-comum, o que foi observado nos resultados.

Infere-se que a aplicação de solução com calcário no sulco de semeadura foi eficiente para aumentar a produtividade de grãos do feijoeiro-comum, em parte pela maior disponibilidade de $\mathrm{P}$, conforme discutido no experimento 1 , mas podendo também ser influenciada por outros fatores (elevação de $\mathrm{pH}$, maior disponibilidade de bases $\mathrm{Ca}, \mathrm{Mg}$ e K), ocasionados pela ação da solução com calcário no sulco de semeadura. Este trabalho é pioneiro no estudo da aplicação de solução com calcário no sulco de semeadura e servirá de ponto de partida para futuras pesquisas sobre esse tema.

\section{CONCLUSÕES}

A aplicação de solução de micropartículas de $\mathrm{CaCO}_{3}$ no sulco de semeadura do feijoeiro-comum causa ligeiro aumento localizado, dos valores de $\mathrm{pH}$, e redução da saturação de Al no solo;

Nas condições em que foram realizados os experimentos, os teores de $\mathrm{Ca}, \mathrm{K}$ e a saturação de bases no solo do sulco de semeadura não são afetados pela solução de micropartículas de $\mathrm{CaCO}_{3}$;

$\mathrm{O}$ uso da solução de micropartículas de $\mathrm{CaCO}_{3}$ no sulco de semeadura proporciona incrementos significativos do número de vagens por área e da produtividade de grãos do feijoeiro-comum.

\section{REFERÊNCIAS}

Alleoni LRF, Cambri MA, Caires EF \& Garbuio FJ (2010) Acidity and aluminum speciation as affected by surface liming in tropical no-till soils. Soil Science Society of America Journal 74:1010-1017.

Bair KE \& Davenport JR (2012) Influence of recent acidification on available phosphorus indices and sorption in Washington State soils. Soil Science Society of America Journal 76:515-521.

Briedis C, Sá JCM, Caires EF, Navarro JF, Inagaki TM, Boer A, Ferreira AO, Quadros Neto C, Canalli LB \& Santos JB (2012) Changes in organic matter pools and increases in carbon sequestration in response to surface liming in an oxisol under long-term-no-till. Soil Science Society of America Journal 76:151-160.

Caires EF, Alleoni LRF, Cambri MA \& Barth G (2005) Surface application of lime for crop grain production under a no-till system. Agronomy Journal, 97:791-798.

Caires EF, Garbuio FJ, Churka S, Barth G \& Correa JCL (2008) Effect of soil acidity amelioration by surface liming on no-till corn, soybean and wheat root growth and yield. European Journal of Agronomy, 28:57-64.

Churka Blum S, Caires EF \& Alleoni LRF (2013) Lime and phosphogypsum application and sulfate retention in subtropical soils under no-till system. Journal of Soil Science and Plant Nutrition, 13:279-300.

Doorenbos J \& Pruitt WO (1976) Crop and water requirements. Rome, FAO. 179p. (FAO Irrigation and Drainage Paper, 24).

Embrapa - Empresa Brasileira de Pesquisa Agropecuária (1997) Manual de métodos de análise de solo. $2^{\mathrm{a}}$ ed. Rio de Janeiro, Embrapa Solos. 212p. (Documentos, 1).

Fageria NK \& Baligar VC (2008) Ameliorating soil acidity of tropical Oxisols by liming for sustainable crop production. Advances in Agronomy, 99:345-399.

Fageria NL, Baligar VC \& Jones CA (2011) Growth and mineral nutrition of field crops. Boca Raton, CRC Press. 586p.

Malavolta E, Vitti GC \& Oliveira SA (1997) Avaliação do estado nutricional das plantas: princípios e aplicações. $2^{\text {nd }}$ ed. Piracicaba, Potafos. 319p.

Mendez J \& Kamprath J (1978) Liming of latosols and the effect on phosphorus response. Soil Science Society of. America Journal, 42:86-88.

Rev. Ceres, Viçosa, v. 62, n.6, p. 597-606, nov-dez, 2015 
Miranda LN, Miranda JCC, Rein TA \& Gomes AC (2005) Lime under notillage and conventional planting systems for soybean and corn in Red Latosol (Oxisol). Pesquisa Agropecuária Brasileira, 40:563-572.

Pagani A \& Mallarino AP (2012) Soil pH and crop grain yield as affected by the source and rate of lime. Soil Science Society of America Journal, 76:1877-1886.

Pelá A, Rodrigues MS, Santana JS \& Teixeira IR (2009) Fontes de fósforo para adubação foliar na cultura do feijoeiro. Scientia Agraria, 10:313 318

Silva EB, Resende JCF \& Cintra WBR (2001) Response of common bean to phosphorus doses in sandy soil. Ciência Rural 31:973-977.

Soratto RP \& Crusciol CAC (2008) Dolomite and phosphogypsum surface application effects on annual crops nutrition and yield. Agronomy Journal 100:261-270.

Souza FS, Faquin V, Torres PRF \& Baliza DP (2006) Liming and organic fertilizer: influence on phosphorus adsorption in soils. Revista Brasileira de Ciência do Solo, 30:975-983.
Souza RF, Faquin V, Lima Sobrinho RR \& Oliveira EAB (2010) Influência de esterco bovino e calcário sobre o efeito residual da adubação fosfatada para a Brachiaria brizantha cultivada após o feijoeiro. Revista Brasileira de Ciência do Solo, 34:143-150.

Valderrama M, Buzetti S, Benett CGS, Andreotti M, Arf O \& Sá ME (2009) Fontes e doses de nitrogênio e fósfor em feijoeiro no sistema plantio direto. Pesquisa Agropecuária Tropical, 39:191-196.

Vieira C, Paula Júnior TJ \& Borém A (2006) Feijão. $2^{a}$ ed. Viçosa, UFV. $500 \mathrm{p}$

Zucareli C, Prando AM, Ramos Junior EU \& Nakagawa J (2011) Phosphorus on the productivity and seed quality of bean Carioca Precoce cultivated during the rainy season. Revista Ciência Agronômica, 42:32-38. 